\title{
Pancake kidney: A rare developmental anomaly
}

\author{
Alok Kumar Tiwari, MS, ${ }^{*}$ Anil Kumar Choudhary, MS, ${ }^{\dagger}$ Hemant Khowal, MBBS, ${ }^{*}$ Poras Chaudhary, MS, ${ }^{*}$ \\ Mohinder. P. Arora, MS
}

*Department of Surgery, Lady Hardinge Medical College, New Delhi, India; †Department of Surgery, Dr Ram Manohar Lohia Hospital, New Delhi, India

Cite as: Can Urol Assoc J 2014;8(5-6):e451-2. http://dx.doi.org/10.5489/cuaj.1933 Published online June 19, 2014.

\section{Abstract}

There are many developmental anomalies of the kidney. Pancake kidney is one of the rarest types of renal ectopia. We report a case of pancake kidney which was detected incidentally while treating a female patient for a urinary tract infection. Although urinary system anomalies often coexist with malformations of other organs and systems, no associated anomalies could be detected in this case. Pancake kidney is usually managed by surgery, but this case was managed conservatively without any complication.

\section{Introduction}

Pancake, disc, shield or doughnut kidney is that which has joined at the medial borders of each pole to produce a doughnut or ring-shaped mass. When there is more extensive fusion along the entire medial aspect of each kidney, a disc or shield shape is created. The pelvis is anteriorly placed and the ureters remain uncrossed. Each collecting system drains its respective half of the kidney and does not communicate with the opposite side. ${ }^{1}$ We present a brief review of its clinical presentation, embryological development and management.

\section{Case report}

A 19-year-old female presented to the outpatient department with chief complaints of lower abdominal pain for 2 months, fever and burning micturition for 15 days. She had no history of pelvic inflammatory disease, menstrual irregularities or other associated comorbidities. Her abdominal examination was unremarkable with clear bilateral renal angles. The routine urine microscopic examination revealed 7 white blood cells/ high power field, although the kidney function test and x-ray KUB (kidney, ureter, bladder) were normal. An ultrasonogra- phy of the KUB region revealed an abnormally located kidney in the bony pelvis as a single fused mass and there were no evidence of calculus, obstruction or hydronephrosis. Contrastenhanced computed tomography (CECT) of the abdomen (Fig. 1 , Fig. 2) revealed a fused mass situated in the pelvic cavity with short course uncrossed ureters opening separately into the urinary bladder. She was given a course of antibiotics based on urine culture and sensitivity test that alleviated her symptoms (pain, fever, burning micturition), but she was followed up regularly to evaluate her kidney function (i.e., serum urea, serum creatinine, blood urea nitrogen, electrolytes).

\section{Discussion}

Wilmer in 1938 was the first to describe the logical categorization of fusion anomalies of the kidney, while McDonald and McClellan in 1957 refined and expanded the classification given by Wilmer. ${ }^{2}$ There are 2 types of renal ectopia: simple renal ectopia and crossed renal ectopia (i.e., crossed ectopia with fusion, crossed ectopia without fusion, solitary crossed ectopia and bilateral crossed ectopia).

A recent review of prenatal ultrasonography detected an incidence of $0.003 \%$ of crossed renal ectopia in India. The incidence of renal ectopia is 1 in 400 cases of autopsy and $85 \%$ of these occur with a fused kidney. ${ }^{3}$ The most common type of renal ectopia is horseshoe kidney, with an incidence of 1 in every 700 autopsies. ${ }^{4}$ Pancake kidney is a very rare type of fused renal ectopia and its exact incidence is unknown. Looney and Dodd were the first to define and describe the pancake kidney. ${ }^{5}$ The pancake kidney is more common in men, although it can be discovered in any age group, and is often associated with other genitourinary and vertebral anomalies. Pancake kidney is described as a renal mass located in the pelvis which is formed by complete medial fusion of renal parenchyma without intervening septum. Each kidney has its own collecting system and anteriorly placed short ureters entering the bladder normally.

The presence of a pancake kidney may predispose the patient to recurrent urinary tract infections and the forma- 

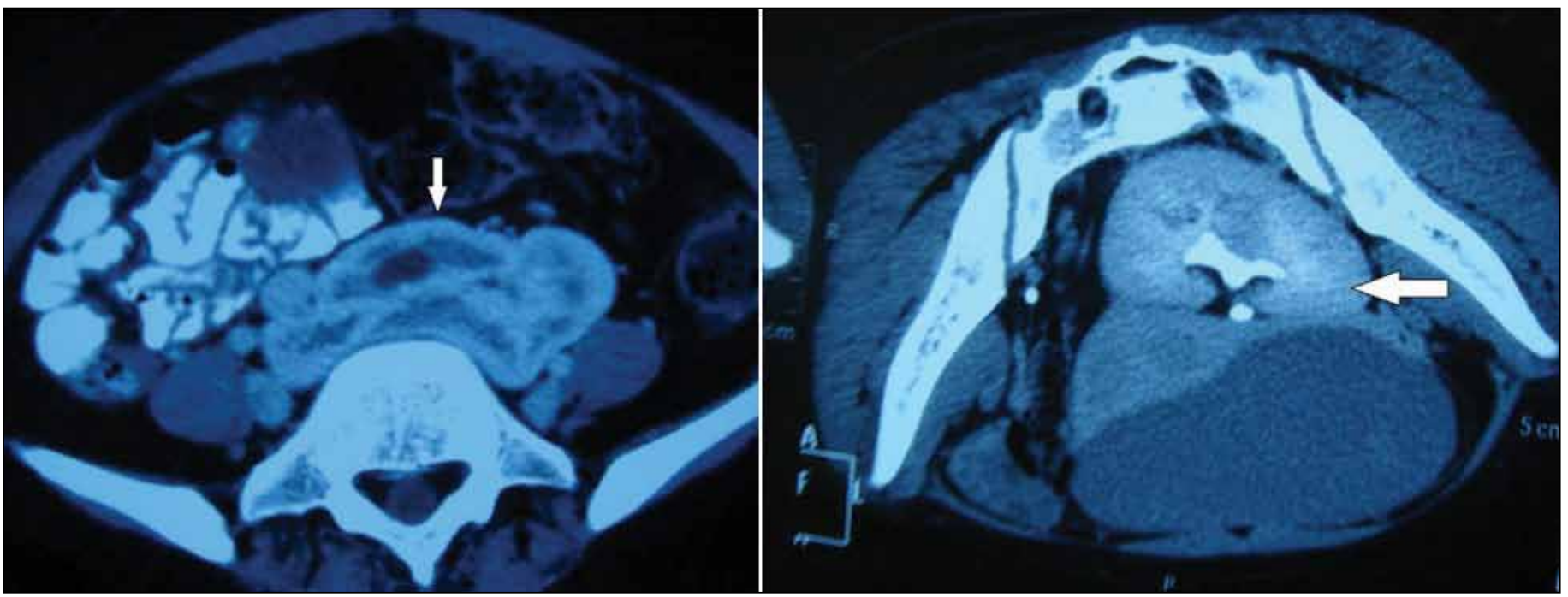

Fig. 1. A contrast-enhanced computed tomography abdomen scan showing the centrally placed pancake kidney.

tion of stones due to the probable rotation anomaly of the collecting system and short ureters which are prone to stasis and obstruction. Patients with pancake kidney are usually asymptomatic, but may present with features of urinary tract infection, fever and vague lower abdominal pain. Sometimes patients may present with extra renal symptoms, such as amenorrhea, failure to conceive or iliac vessel aneurysm. The diagnosis is always incidental. In earlier days, excretory urography was the usual method of detection, which is now replaced by ultrasonography, CT and radionucleotide scanning. The presence of pancake kidney does not mean that the patient will have progressive renal failure. If there are symptoms of renal failure, deranged renal function test

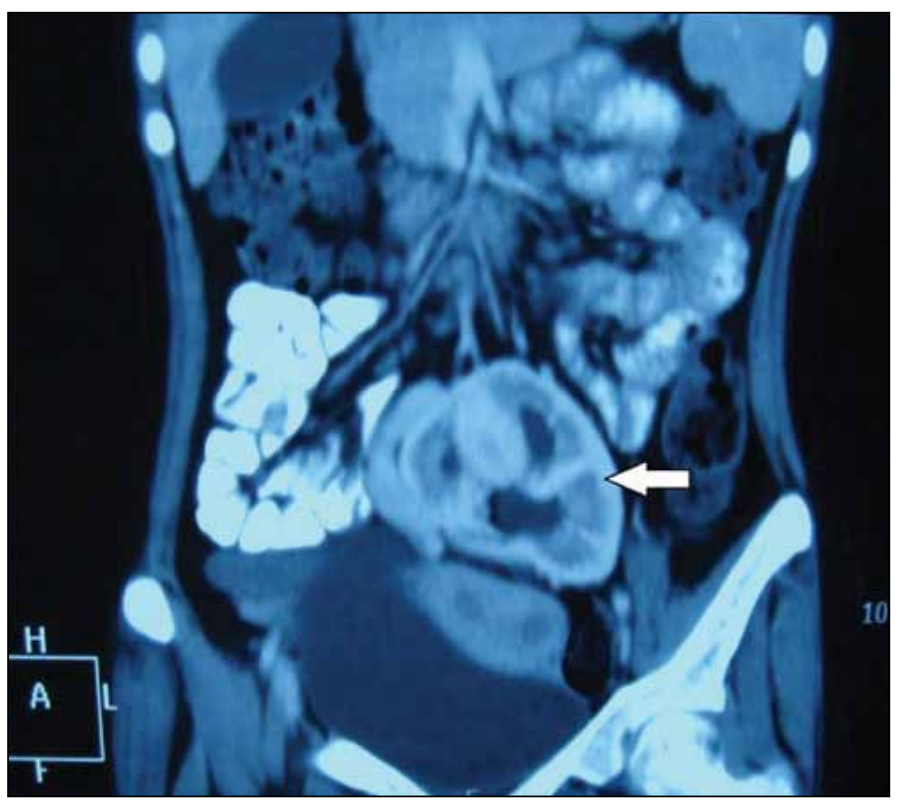

Fig. 2. A contrast-enhanced computed tomography abdomen scan in the coronal section showing the pancake kidney placed in the pelvis with short ureters (arrow). or features of urinary outflow obstruction or obstructive uropathy, surgery is warranted. Division of the parenchyma may lead to renal vascular damage, necrosis of the kidney, infarction of the kidney or postoperative renal failure. Asymptomatic cases can be managed conservatively with long-term follow-up of renal function, which can help detect complications, such as urinary tract infection, calculi and obstruction. In our case we managed the patient conservatively with regular follow-up. We avoided unnecessary investigations and extensive surgery on pancake kidney.

\section{Conclusion}

Our patient was managed conservatively. More research is needed to establish standards for the management of pancake kidney.

Competing interests: Dr. Tiwari, Dr. Choudhary, Dr. Khowal, Dr. Chaudhary and Dr. Arora all declare no competing financial or personal interests.

This paper has been peer-reviewed.

\section{References}

1. Glenn Jf. Fused pelvic kidney. J Urol 1958;80:7-9.

2. Wein AJ editor. Campbell-Walsh Urology. 9th edition. China; 2007:3283-9.

3. Shoemaker R, Braasch WF. Fused kidneys. J Urol 1939;41:1-7.

4. Eisendrath DN, Phifer FM, Culver HB. Horseshoe kidney. Ann Surg 1925;82:735-64. http://dx.doi. org/10.1097/00000658-192511010-00009

5. Looney WW, Dodd DL. An ectopic (pelvic) completely fused (cake) kidney associated with various anomalies of the abdominal viscera. Ann Surg 1926;84:522-4.

Correspondence: Dr. Alok Kumar Tiwari, Department of Surgery, Lady Hardinge, Medical College and associated Dr Ram Manohar Lohia Hospital, New Delhi, India; draloktyl@gmail.com 